\title{
Fibrinolysis and atherogenesis in the JCR:LA-cp rat in relation to insulin and triglyceride concentrations in blood
}

\author{
D.J.Schneider ${ }^{1}$, P.M. Absher ${ }^{1}$, D. Neimane ${ }^{1}$, J.C. Russell ${ }^{2}$, B. E. Sobel ${ }^{1}$ \\ ${ }^{1}$ Department of Medicine, University of Vermont College of Medicine, Burlington, Vermont, USA \\ ${ }^{2}$ Department of Surgery, University of Alberta, Edmonton, Alberta, Canada
}

\begin{abstract}
Summary Increased concentrations of plasminogen activator inhibitor type-1 (PAI-1) in blood and attenuated fibrinolytic activity, hypertriglyceridaemia, and insulin resistance are common in subjects with obesity and non-insulin-dependent diabetes mellitus who are at markedly increased risk for coronary artery disease. To clarify potentially causal relationships between these phenomena, we studied JCR:LA-cp rats, animals that are insulin resistant and prone to vasculopathy. Blood and aortas were obtained from lean and corpulent animals at 1, 2, 4, 6, and 9 months of age. The homozygous corpulent rats were hyperinsulinaemic and hypertriglyceridaemic at all ages tested. Increased activity of PAI-1 was pre-
\end{abstract}

sent in blood from corpulent animals at 1, 6, and 9 months of age. Positive correlations were observed between blood PAI-1 and both insulin and triglycerides. As judged from results with aortic rings in in vitro culture, the increased PAI-1 in blood was anteceded by increased expression of PAI-1 in arterial walls. Thus, changes indicative of inhibition of the fibrinolytic system capacity precede gross atherosclerosis. [Diabetologia (1998) 41: 141-147]

Keywords Atherosclerosis, diabetes, hyperinsulinaemia, hypertriglyceridaemia, plasminogen activator inhibitor-1.
Fibrinolytic system proteins in blood and arterial walls [1-6] have been implicated in atherogenesis and in the macroangiopathy typical of non-insulindependent diabetes mellitus (NIDDM). Impairment of fibrinolysis may lead to accumulation of microthrombi that may induce or exacerbate atherogenesis. High concentrations of plasminogen activator inhibitor type-1 (PAI-1) and attenuated fibrinolytic activity are seen in human subjects with obesity, NIDDM, or both [7-10]. Obesity and particularly early

Received: 8 July 1997 and in revised form: 23 September 1997

Corresponding author: Dr. P. M. Absher, Department of Medicine, Given Building, Room C-323, University of Vermont College of Medicine, Burlington, VT 05405, USA

Abbreviations: PAI-1, Plasminogen activator inhibitor-1; t-PA, tissue plasminogen activator; IRI, immunoreactive insulin; HBSS, Hank's balanced salt solution; DME/F12, Dulbecco's modified Eagle's medium with Ham's nutrient mixture F12; BSA, bovine serum albumin; NIDDM, non-insulin-dependent diabetes mellitus. stage NIDDM are also characterized by increased concentrations of immunoreactive insulin (IRI) evident in plasma. Several observations suggest a causal connection between hyperinsulinaemia, insulin resistance and impaired fibrinolysis in blood [7-10]. However, the temporal relationships between hyperinsulinaemia, hypertriglyceridaemia, and impaired fibrinolytic system capacity secondary to increased PAI-1 in blood and the onset of macroangiopathy have not yet been elucidated. Accordingly, we studied JCR:LA-cp (corpulent) rats, animals genetically predisposed to develop severe insulin resistance with hyperinsulinaemia, hyperlipidaemia, obesity, and subsequent macroangiopathy [11-14].

The JCR:LA-cp atherosclerosis-prone rat strain is one in which many aspects of early NIDDM and atherogenesis are simulated [11-14]. This strain incorporates an autosomal recessive cp (corpulent) gene and JCR:LA-cp rats homozygous for the $\mathrm{cp}$ gene (cp/ $\mathrm{cp}$ ) are obese, insulin resistant, and hypertriglyceridaemic [13]. Heterozygous ( $+/ \mathrm{cp})$ and homozygous 
normal $(+/+)$ rats are lean and indistinguishable from members of the parent LA/N strain. JCR:LA$\mathrm{cp} \mathrm{cp} / \mathrm{cp}$ rats are detectably obese by 3 weeks of age and insulin resistant by 8 weeks of age. In the male $\mathrm{cp} / \mathrm{cp}$ rat glucose uptake and turnover are no longer responsive to insulin by 12 weeks of age [12]. Marked VLDL hyperlipidaemia is evident and attributable to increased VLDL secretion rather than reduced clearance [13]. The male JCR:LA-cp rat develops atherosclerosis with frank lesions usually beginning after 6 months of age and extensive lesions evident in the aortic arch in $100 \%$ of male cp/cp rats at 9 months of age [14]. A less severe form of atherosclerosis is manifested after 18 months of age in female JCR:LA-cp rats homozygous for the corpulent trait [14]. Thus, the JCR:LA-cp rat provides a unique opportunity to characterize the changes in fibrinolytic activity in blood in temporal relation to changes in concentrations of moieties such as insulin and triglyceride. Because the vasculopathy is predominant in the male animals, male JCR:LA-cp homozygous corpulent (cp/ cp) and homozygous control $(+/+)$ rats of $1,2,4,6$, and 9 months of age were studied. Female homozygous corpulent $(\mathrm{cp} / \mathrm{cp})$ and control $(+/+)$ rats of 6 months of age were studied.

\section{Materials and methods}

Animals were handled in accordance with the National Institutes of Health (NIH) guidelines for laboratory animal care (Principles of laboratory animal care, NIH publication No. 85-23) and with protocols approved by the University of Vermont Animal Care and Use Committee. Animals were kept on a $12 \mathrm{~h}$ light/dark cycle and fed ad libitum. Samples of blood and tissue were obtained from animals in a fed state. Rats were anaesthetized with intraperitoneal injections of sodium pentobarbital (120 mg/kg body weight). Blood was obtained by cardiac puncture and the aorta was removed under sterile conditions. Blood samples were centrifuged for $20 \mathrm{~min}$ at $2000 \mathrm{~g}$ at $4{ }^{\circ} \mathrm{C}$. Plasma was collected and stored at $-70^{\circ} \mathrm{C}$. The numbers of JCR:LA-cp male, cp/cp corpulent and $+1+$ lean rats used in the experiments were: at $1(n=3 \mathrm{cp}, 3+/+), 2(n=3 \mathrm{cp}$, $3+/+), 4(n=4 \mathrm{cp}, 4+/+), 6(n=7 \mathrm{cp}, 8+/+)$, and 9 $(n=10 \mathrm{cp}, 6+/+)$ months of age; where $n=$ number rats in each experimental group. At 6 months of age there were $7 \mathrm{fe}-$ male $\mathrm{cp} / \mathrm{cp}$ rats and 8 female $+/+$ rats.

Quantification of plasma glucose, insulin, and triglycerides. Plasma glucose was measured by the glucose oxidase method of Trinder [15] with the use of Sigma kit 315 (Sigma Chemical Co., St. Louis, Mo., USA). Insulin was measured with the use of a Coat-A-Count Insulin Kit TKIN1 (Diagnostic Products Corporation, Los Angeles, Calif., USA). In the assay ${ }^{125} \mathrm{I}$-labelled insulin competes with insulin in the plasma sample for sites on insulin-specific antibody immobilized to the wall of polypropylene tubes. The antibody-bound fraction was detected with a gamma counter, with the counts being directly proportional to the amount of insulin in the plasma sample. Cross-reactivity with proinsulin is approximately $40 \%$ (per manufacturer's specification). Rat insulin (Crystal Chem, Chicago, Ill., USA) was used to define a standard curve.
Plasma triglycerides were measured by a modification of the method of Bucolo and David [16] with the use of Sigma kit 336. In this assay, triglycerides are converted to glycerol and fatty acids. Through a series of reactions involving ATP and NAD, NADH is generated and detected with a chromogenic substrate, 2-(p-iodophenyl)-3-p-nitrophenyl-5-phenyltetrazolium that forms a highly coloured formazan with a maximum absorbance at $500 \mathrm{~nm}$ directly proportional to the concentration of triglycerides in the sample.

Organ culture. The aortas excised under sterile conditions were placed in sterile Hank's balanced salt solution (HBSS) containing $300 \mathrm{U} / \mathrm{ml}$ penicillin and $300 \mu \mathrm{g} / \mathrm{ml}$ streptomycin. Adherent fat and connective tissue were trimmed and blood cells were washed away with HBSS. The aorta was then cut into ring segments of 1-2 mm widths and cultured in Dulbecco's Modified Eagle's Medium with Ham's nutrient mixture F12 (DME/F12, [Gibco, BRL, Grand Island, N. Y., USA]) supplemented with $1 \%$ bovine serum albumin (BSA, [Sigma]), 50 $\mathrm{U} / \mathrm{ml}$ penicillin, and $50 \mu \mathrm{g} / \mathrm{ml}$ of streptomycin; incubated at $37^{\circ} \mathrm{C}$ in an atmosphere of $5 \% \mathrm{CO}_{2}: 95 \%$ air. Culture medium was exchanged daily. No evidence of infection was observed by microscopic evaluation of all samples. Conditioned media were screened for endotoxin contamination with the Limulus ameobocyte lysate assay and did not exhibit contamination exceeding $0.01 \mathrm{ng} / \mathrm{ml}$ (100-times less than concentrations required to stimulate expression of PAI-1 [17]).

Quantification of PAI-1 and $t$-PA. PAI-1 activity in plasma was measured as previously described [18]. Briefly, a fixed amount of t-PA was added in excess to plasma rapidly generating an inactive complex of t-PA with PAI-1 present in the plasma sample. After dilution, the sample was exposed to plasminogen in the presence of cyanogen bromide fragments of fibrinogen. The amount of plasmin generated was determined based on measurement of the amidolytic activity of plasmin on the chromogenic substrate S-2251 (Chromogenix) resulting in the release of p-nitroaniline determined by absorbance at $405 \mathrm{~nm}$. The amount of residual t-PA activity determines the amount of plasmin formed and is inversely proportional to the amount of PAI-1 activity in the sample.

Plasma t-PA activity was measured with an indirect amidolytic method involving the activation of plasminogen in the presence of fragments of fibrinogen based on generation of para-nitroanilide from the plasmin specific substrate S-2251 (Chromogenix) [19].

PAI-1 protein content of culture medium obtained from aorta ring cultures was assayed by Western blot analysis. PAI1 was immunoprecipitated from the conditioned medium by overnight incubation at $4{ }^{\circ} \mathrm{C}$ with a rabbit anti-rat $\mathrm{IgG}$. The antibody/antigen complexes were isolated with protein $\mathrm{G}$ sepharose (Pharmacia, Piscataway, N.J., USA). The samples were electrophoresed with SDS-PAGE under non-reducing conditions in order to prevent separation of the IgG into light and heavy chains. After transfer of proteins to Trans-Blot membrane (Bio-Rad, Hercules, Calif., USA), PAI-1 was detected with rabbit anti-rat PAI-1 IgG (American Diagnostica, Greenwich, Conn., USA) and a secondary alkaline phosphatase conjugated antibody mouse anti-rabbit IgG. Gels were scanned with a Logitech Scanman (Logitech, Inc., Fremont, Calif., USA) with the use of a Sigma Gel program (Jandel Corporation, San Rafael, Calif., USA) for calculation of intensity of standard PAI-1 and PAI-1 present in samples. In order to facilitate a semiquantitative analysis of each blot, a fixed amount of a rat PAI-1 standard (American Diagnostica) was electrophoresed on each gel. Thus, the intensity of PAI-1 in a given sample is expressed as a fraction of the intensity of the PAI-1 standard. 

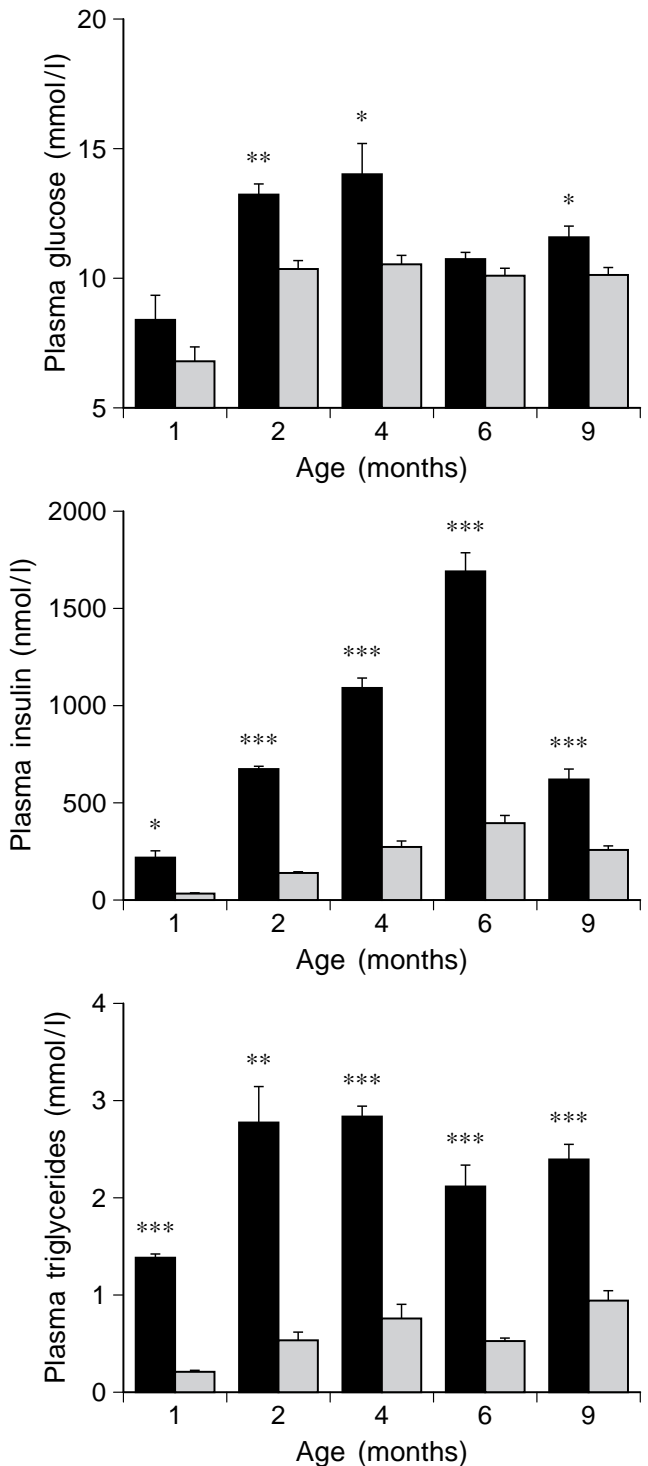

Fig. 1. Concentrations of glucose, insulin, and triglycerides in blood obtained from JCR:LA-cp male, cp/cp corpulent and $+1+$ lean 1 rats at $1(n=3 \mathrm{cp}, 3+1+), 2(n=3 \mathrm{cp}, 3+1$ $+), 4(n=4 \mathrm{cp}, 4+/+), 6(n=7 \mathrm{cp}, 8+/+)$, and $9(n=10 \mathrm{cp}$, $6+1+)$ months of age; $(\mathrm{n}=$ number rats in each experimental group). Data are means \pm SEM of plasma concentrations of glucose, insulin, and triglycerides. Statistical differences were determined by $t$-test for samples with equal variances and by Mann-Whitney Rank Sum Test for samples with unequal variances. Statistical tests compared $\mathrm{cp} / \mathrm{cp}$ rats with $+/+$ rats at each observation interval, $* p<0.05, * * p<0.01, * * * p<0.001$

Statistical analysis. Student's $t$-test was used to detect differences between experimental groups for samples having equal variances. The Mann-Whitney Rank Sum Test was used for samples with unequal variances. Spearman's Rank Order Correlation was used for correlation tests.

\section{Results}

Plasma glucose, insulin, and triglycerides. Figure 1 shows the concentrations of plasma glucose, insulin
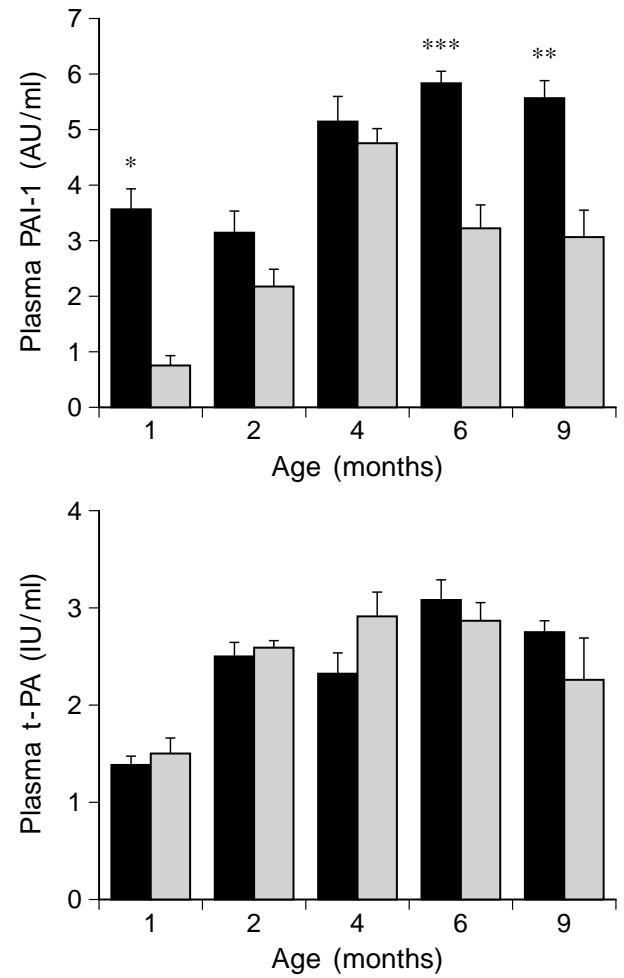

Fig. 2. PAI-1 and t-PA activity in blood obtained from JCR:LA-cp male, cp/cp corpulent $\square$ and $+/+$ lean $\square$ rats at $1(n=3 \mathrm{cp}, 3+/+), 2(n=3 \mathrm{cp}, 3+1+), 4(n=3 \mathrm{cp}, 4+/+), 6$ $(n=7 \mathrm{cp}, 8+/+)$, and $9(n=10 \mathrm{cp}, 6+/+)$ months of age; ( $n=$ number rats in each experimental group). Data are means \pm SEM of plasma concentrations of PAI- 1 and t-PA. PAI- 1 is expressed in arbitrary units (AU) indicating measurement of PAI-1 remaining after the reaction. t-PA is expressed in international units (IU) and is a direct measure of t-PA concentration detected by the assay. Statistical differences were determined by $t$-test for samples with equal variances and by Mann-Whitney Rank Sum Test for samples with unequal variances. Statistical tests compared $\mathrm{cp} / \mathrm{cp}$ rats with $+/+$ rats at each observation interval, $* p<0.05, * * p<0.01$, *** $p<0.001$

and triglycerides in JCR:LA-cp $\mathrm{cp} / \mathrm{cp}$ corpulent and $+/+$ lean control rats at $1,2,4,6$, and 9 months of age. Glucose was elevated in $\mathrm{cp} / \mathrm{cp}$ rats compared with $+/+$ controls at 2,4 , and 9 months of age. Insulin and triglyceride were markedly elevated at all observation times.

PAI-1 activity in rat plasma. As shown in Figure 2, PAI-1 activity in plasma of JCR:LA-cp cp/cp corpulent rats was significantly elevated compared with that in $+1+$ lean control rats in animals of 1,6 , and 9 months of age. Although plasma concentrations of t-PA appeared to be increased in both $\mathrm{cp} / \mathrm{cp}$ corpulent and $+/+$ lean control rats at 2 to 9 months of age, there were no significant differences between $\mathrm{cp} / \mathrm{cp}$ and + / + rats.

Correlation of PAI-1 with plasma glucose, insulin, and triglycerides. The increased PAI-1 activity in plasma 

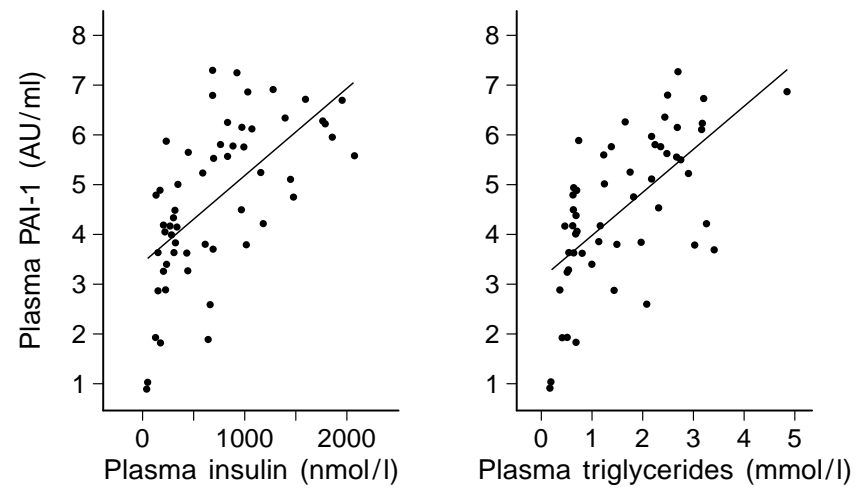

Fig. 3. Correlation between plasma PAI-1 and insulin and triglycerides. Data from 50 male and 12 female JCR:LA-c cp/cp and $+/+$ rats at $1(n=6), 2(n=6), 4(n=6), 6(n=15$ male, 12 female $)$, and $9(n=16)$ months of age are pooled. Increased PAI-1 activity in plasma from JCR:LA-cp rats was significantly correlated with increases in insulin $(r=0.659, p<0.001)$ and with increases in triglycerides $(r=0.651, p<0.001)$. Data analysed by Spearman's Rank Order Correlation

was significantly correlated with increases in insulin $(p<0.001)$ and with increases in triglycerides $(p<0.001)$ (Fig. 3), whereas PAI-1 activity was not correlated with glucose concentration (data not shown).

Comparison of results in male and female rats of 6 months of age. As shown in Figure 4, plasma glucose concentrations did not differ among any of the groups. Both male and female $\mathrm{cp} / \mathrm{cp}$ rats had significantly increased concentrations of insulin in plasma compared with values in $+/+$ control rats, $p<0.001$. Insulin values in male $\mathrm{cp} / \mathrm{cp}$ rat plasma were not different from those in female $\mathrm{cp} / \mathrm{cp}$ rat plasma, while male $+/+$ rats had slightly higher insulin values compared with $+/+$ females $(p<0.05)$. Both male and female $\mathrm{cp} / \mathrm{cp}$ rats showed significantly increased concentrations of triglycerides in plasma (Fig. 4). The increased plasma triglyceride concentrations in female $\mathrm{cp} / \mathrm{cp}$ rats $(7.12 \pm 0.60 \mathrm{mmol} / \mathrm{l})$ were more pronounced than in male cp/cp rats $(2.09 \pm 0.22 \mathrm{mmol} / \mathrm{l})$. Plasma PAI-1 activity in both male and female $\mathrm{cp} / \mathrm{cp}$ rats was higher than in corresponding $+/+$ rats (Fig.5). There was no difference in PAI-1 activity in male compared with female $\mathrm{cp} / \mathrm{cp}$ rats, whereas female $+1+$ rats had moderately higher PAI-1 activity compared with male $+/+$ rats $(p<0.02)$. No differences in t-PA activity were seen.

PAI-1 protein in aorta ring culture medium. Results of Western blot analysis for PAI-1 protein accumulated in 24-h conditioned media from aortic rings in culture from 6-month-old male and female $\mathrm{cp} / \mathrm{cp}$ and $+/$ + rats are shown in Figure 6. As can be seen, the elaboration of PAI-1 over $24 \mathrm{~h}$ was substantially (4-fold) and significantly $(p<0.001)$ higher in aortas taken from corpulent males than from lean males and fe-
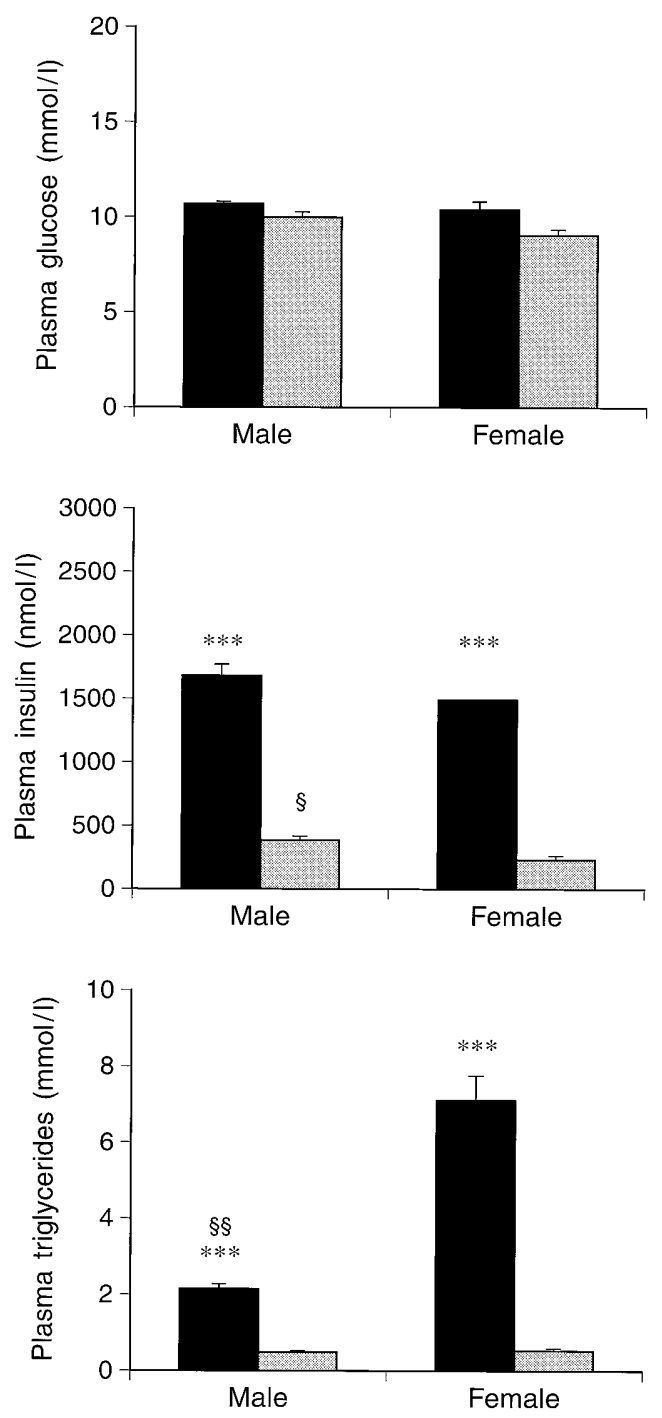

Fig.4. Comparison of glucose, insulin, and triglyceride concentrations in blood obtained from JCR:LA-cp cp/cp corpulent male $(n=7)$ and female $(n=7) \square$, and $+/+$ lean male $(n=8)$ and female $(n=8) \square$ rats at 6 months of age. Data are means \pm SEM of plasma concentrations of glucose, insulin, and triglycerides. Statistical differences were determined by $t$ test for samples with equal variances and by Mann-Whitney Rank Sum Test for samples with unequal variances. Statistical significance comparing $\mathrm{cp} / \mathrm{cp}$ rats with $+/+$ rats; $* * *$ $p<0.001$, and comparing male $\mathrm{cp} / \mathrm{cp}$ rats with female $\mathrm{cp} / \mathrm{cp}$ rats; $\S p<0.05, \S \S p<0.01$

males of both phenotypes. Although the semiquantitative analysis precluded determination of subtle differences, there was a consistent and marked excess of PAI-1 seen in media conditioned by aortas from corpulent males.

\section{Discussion}

Fibrinolytic capacity reflects a balance between activity of plasminogen activators in blood (primarily 

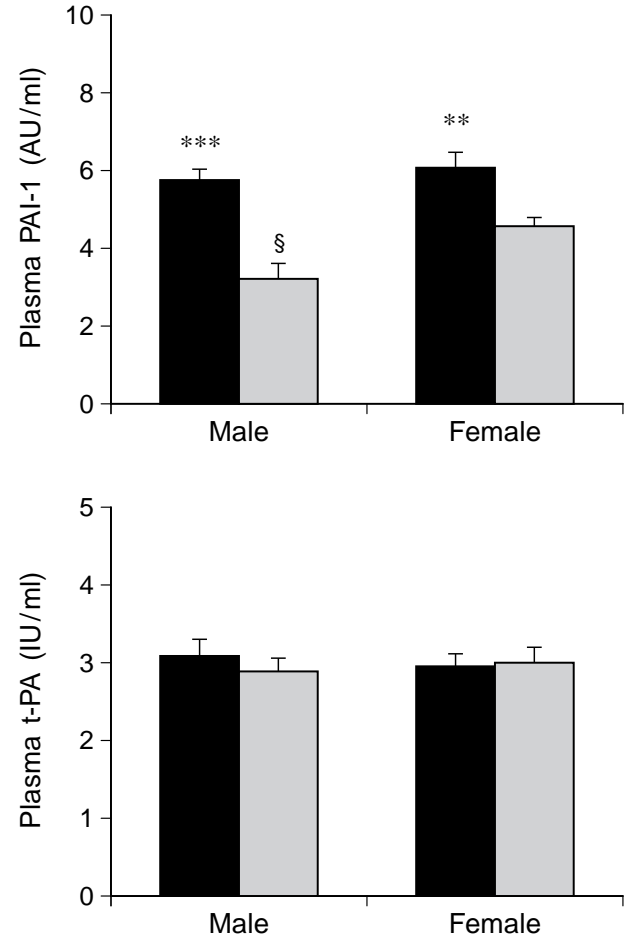

Fig. 5. Comparison of PAI-1 and t-PA activity in blood obtained from JCR:LA-cp cp/cp corpulent male $(n=7)$ and female $(n=7) \square$, and $+/+$ lean male $(n=8)$ and female $(n=8)$ rats at 6 months of age. Data are means \pm SEM of plasma concentrations of PAI-1 and t-PA. Statistical differences were determined by $t$-test comparing $\mathrm{cp} / \mathrm{cp}$ rats with $+/+$ rats and male rats with female rats. ** $p<0.01$, *** $p<0.001$ for $\mathrm{cp} / \mathrm{cp}$ rats compared with $+/+$ rats, $\S p<0.02$ for male rats compared with female rats

t-PA) and their primary physiologic inhibitor, PAI-1. It is likely to be critical in influencing the thrombotic response to vascular injury and plaque rupture. Decreased fibrinolytic capacity is anticipated to potentiate the persistence of microthrombi. The net effect is likely to be increased arterial mural exposure to thrombi and their associated mitogens that can accelerate atherogenesis by potentiating migration and proliferation of smooth muscle cells as well as deposition of extracellular matrix [20-22].

Subjects with NIDDM have a high incidence of cardiovascular events [23-25]. Up to $16.6 \%$ of patients will have a cardiovascular event during the first 10 years after diagnosis of diabetes [23]. This high incidence of cardiovascular events early after the diagnosis of diabetes suggests that the insulin resistant state precedes the diagnosis of diabetes by many years and is likely to contribute. Hyperinsulinaemia secondary to end-organ insulin resistance is present 1 to 2 decades before the onset of overt diabetes [26]. We, and others have shown that the concentration and activity of PAI-1 are increased in the blood of subjects with NIDDM and obesity [7-10].

JCR:LA-cp rats develop insulin resistance and resultant hyperinsulinaemia, hypertriglyceridaemia,

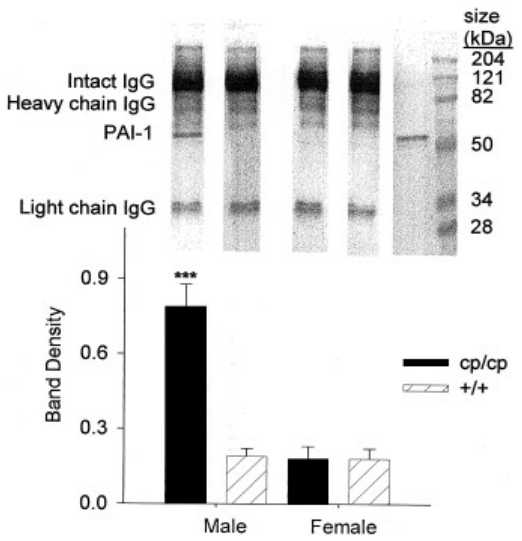

Fig. 6. PAI-1 protein accumulated in $24 \mathrm{~h}$ conditioned media of cultured aorta rings obtained from JCR:LA-cp cp/cp corpulent male $(n=7)$ and female $(n=8), \square$ and $+1+$ lean male $(n=7)$, and female $(n=8) \square$ rats at 6 months of age. Western blot shows bands corresponding to graph below and PAI-1 standard (far right). Data are means \pm SEM of concentrations of PAI-1 in conditioned media. Statistical differences were determined with the use of the Mann-Whitney Rank Sum Test for comparison of $\mathrm{cp} / \mathrm{cp}$ rats with $+/+$ rats, $* * * p<0.001$

and atherosclerosis [11-14], features consistent with NIDDM and its antecedents. Accordingly, we characterized the temporal relationship between the development of abnormalities in metabolism and fibrinolytic capacity in blood in these animals.

Our results show that the activity of PAI-1 is increased in the blood of the cp/cp rats at 1, 6, and 9 months of age. The increased PAI-1 activity was observed in blood from 1-month-old animals homozygous for the corpulent trait. Because previous studies with this animal preparation have indicated that raised intimal lesions are not present (by electron microscopy) in male animals less than 6 months of age [14], the abnormal expression of PAI-1 precedes the development of vasculopathy. Female rats homozygous for the corpulent gene do not develop significant atherosclerosis until 18 months of age [14]. Thus, increased PAI-1 activity in blood precedes the development of vasculopathy in both sexes. The activity of PAI-1 is increased similarly in the blood of male and female corpulent rats. Despite this, the development of vasculopathy is delayed in females. These results are consistent with the relatively delayed development of atherosclerosis in women with NIDDM [23, 25 ] and underscore its multifactorial aetiology.

Oestrogen appears to have a protective effect on the development of vasculopathy $[27,28]$. The cardiovascular protection has been described as a direct effect on the vessel wall as well as an indirect effect on lipid metabolism [27, 28]. Wagner and colleagues [28] have shown that although combined therapy with oestrogen and progesterone may increase fasting insulin and glucose levels in cynomolgus monkeys, it does not appear to accelerate the progression of vasculopathy. Accordingly the slower progression 
of vasculopathy in the female corpulent JCR:LA-cp rat compared with the corpulent male may be related in part to the effects of oestrogen.

As in human subjects [7-10], activity of PAI-1 in blood from JCR:LA-cp rats correlated positively and significantly with concentrations of both insulin and triglycerides in blood. No significant association was observed between the modest elevations in glucose and the increased PAI-1 activity seen in these animals. These results are consistent with our results in vitro demonstrating direct effects of both insulin and triglycerides on expression of PAI- 1 and a synergistic augmentation when increased concentrations of both agents are present [29]. Nevertheless, the male and female cp/cp rats exhibited similar elevations of insulin and of PAI-1 activity but triglycerides were significantly increased in female $\mathrm{cp} / \mathrm{cp}$ compared with male $\mathrm{cp} / \mathrm{cp}$. Results in JCR:LA-cp rats are consistent with the hypothesis that hormonal and metabolic abnormalities that precede the development of overt diabetes may potentiate the development of atherosclerosis, in part, by modulating expression of fibrinolytic capacity.

The cellular source of increased PAI-1 in the blood of subjects with obesity and NIDDM has not yet been elucidated. Data from studies in vivo and in vitro suggest that hepatocytes, adipocytes, platelets and vascular wall cellular components can contribute [29-32]. Arterial expression of PAI-1 is increased in rabbits infused with insulin and proinsulin [33]. We have shown that insulin directly augments expression of PAI- 1 by human arteries in vitro [34]. Our results with aortas obtained from JCR:LA-cp corpulent rats support the possibility that increased arterial expression of PAI-1 may contribute to the increased PAI-1 in blood from corpulent males. Thus, increased accumulation of PAI-1 was observed in media of cultured aortic rings from $\mathrm{cp} / \mathrm{cp}$ males even without addition of exogenous insulin. The lack of increased expression of PAI-1 by aortic rings from corpulent females is consistent with decreased vascular elaboration of PAI-1 in direct proportion to the circulating concentration of oestrogen, as reported in the Framingham Offspring Study [35].

Both the hyperinsulinaemia and hypertriglyceridaemia seen in corpulent atherosclerosis-prone JCR:LA-cp rats of 4 weeks of age or more are paralleled by increased PAI-1 activity in blood. These abnormalities precede the development of atherosclerosis. Further definition of the role of PAI-1 in atherogenesis may delineate potential targets for therapy designed to retard progression of macroangiopathy.

\section{References}

1. Schneider DJ, Nordt TK, Sobel BE (1993) Attenuated fibrinolysis and atherogenesis in type II diabetic subjects. Diabetes 42: 1-7

2. Negri M, Sheiban I, Arigliano PL, et al. (1993) Interrelation between angiographic severity of coronary artery disease and plasma levels of insulin, C-peptide and plasminogen activator inhibitor-1. Am J Cardiol 72:397-401

3. Chomiki N, Henry M, Alessi MC, Anfosso F, Juhan-Vague I (1994) Plasminogen activator inhibitor-1 expression in human liver and healthy or atherosclerotic vessel walls. Thromb Haemostas 72:44-53

4. Lupu F, Bergonzelli GE, Heim DA, et al. (1993) Localization and production of plasminogen activator inhibitor-1 in human healthy and atherosclerotic arteries. Arterioscler Thromb 13: 1090-1100

5. Padró T, Emeis JJ, Steins M, Schmid KW, Kienast J (1995) Quantification of plasminogen activators and their inhibitors in the aortic vessel wall in relation to the presence and severity of atherosclerotic disease. Arterioscler Thromb Vasc Biol 15:893-902

6. Schleef RR, Loskutoff DJ, Podor TJ (1991) Immunoelectron microscopic localization of type 1 plasminogen activator inhibitor on the surface of activated endothelial cells. J Cell Biol 113: 1413-1423

7. Vague P, Juhan-Vague I, Aillaud MF, et al. (1986) Correlation between blood fibrinolytic activity, plasminogen activator inhibitor level, plasma insulin level and relative body weight in normal and obese subjects. Metabolism 35:250-253

8. McGill JB, Schneider DJ, Arfken CL, Lucore CL, Sobel BE (1994) Factors responsible for impaired fibrinolysis in obese subjects and NIDDM patients. Diabetes 43:104 109

9. Landin K, Stigendal L, Eriksson E, et al. (1990) Abdominal obesity is associated with an impaired fibrinolytic activity and elevated plasminogen activator inhibitor-1. Metabolism 39: 1044-1048

10. Jansson JH, Johansson B, Boman K, Nilsson TK (1991) Hypofibrinolysis in patients with hypertension and elevated cholesterol. J Intern Med 229:309-316

11. Dolphin PJ, Stewart B, Amy RM, Russell JC (1987) Serum lipids and lipoproteins in the atherosclerosis prone LA/Ncorpulent rat. Biochim Biophys Acta 919: 140-148

12. Russell JC, Graham S, Hameed M (1994) Abnormal insulin and glucose metabolism in the JCR:LA-corpulent rat. Metabolism 43: 538-543

13. Russell JC, Koeslag DG, Amy RM, Dolphin PJ (1989) Plasma lipid secretion and clearance in hyperlipidaemic JCR:LA-corpulent rats. Arteriosclerosis 9: 869-876

14. Russell JC, Amy RM (1986) Early atherosclerotic lesions in a susceptible rat model: the LA/N-corpulent rat. Atherosclerosis 60: 119-129

15. Trinder P (1969) Determination of blood glucose using an oxidase-peroxidase system with a non-carcinogenic chromogen oxygen acceptor. J Clin Path 22: 158-161

16. Bucolo, G, David H (1973) Quantitative determination of serum triglycerides by the use of enzymes. Clin Chem 19: 476-487

17. Sawdey M, Podor TJ, Loskutoff DJ (1989) Regulation of type 1 plasminogen activator inhibitor gene expression in cultured bovine aortic endothelial cells. J Biol Chem 264: 10396-10401

18. Chmielewska J, Wiman B (1986) Determination of tissue plasminogen activator and its "fast" inhibitor in plasma. Clin Chem 32:482-485 
19. Angles-Cano E (1984) A spectrophotometric solid-phase fibrin-tissue plasminogen activator activity assay (SOFIAt-PA) for high-fibrin-affinity tissue plasminogen activators. Anal Biochem 153:201-210

20. Ellis V, Dano K (1992) The urokinase receptor and the regulation of cell surface plasminogen activation. Fibrinolysis 6(S4):27-32

21. He CS, Wilhelm SM, Pentland AP, et al. (1989) Tissue cooperation in a proteolytic cascade activating human interstitial collagenase. Proc Natl Acad Sci USA 86:2632-2637

22. Chapman HA Jr, Stone OL (1989) Co-operation between plasmin and elastase degradation by intact murine macrophages. Biochem J 222: 721-725

23. Uusitupa MIJ, Niskanen LK, Siitonen O, Voutilainen E, Pyorala K (1993) Ten-year cardiovascular mortality in relation to risk factors and abnormalities in lipoprotein composition in type 2 (non-insulin-dependent) diabetic and nondiabetic subjects. Diabetologia 36:1175-1184

24. Kannel WB, D'Agostino RB, Wilson PW, Belanger AJ, Gagnon RR (1990) Diabetes, fibrinogen, and risk of cardiovascular disease: the Framingham experience. Am Heart J 120: 672-676

25. Ronnemaa T, Laakso M, Puukka P, Kallio V, Pyorala K (1988) Atherosclerotic vascular disease in middle aged insulin-treated, diabetic patients: association with endogenous insulin secretion capacity. Arteriosclerosis 8:237-240

26. Warram JH, Martin BC, Krolewski AS, Soeldner JS, Kahn CR (1990) Slow glucose removal rate and hyperinsulinaemia precede the development of type II diabetes in the offspring of diabetic parents. Ann Int Med 113: 909-915

27. Farhat MY, Lavigne MC, Ramwell PW (1996) The vascular protective effects of estrogen. FASEB J 10:615-624
28. Wagner JD, Martino MA, Jayo MJ, Anthony MS, Clarkson TB, Cefalu WT (1996) The effects of hormone replacement therapy on carbohydrate metabolism and cardiovascular risk factors in surgically postmenopausal cynomolgus monkeys. Metabolism 45:1254-1262

29. Schneider DJ, Sobel BE (1996) Synergistic augmentation of expression of PAI-1 induced by insulin, VLDL, and fatty acids. Coronary Artery Disease 7:813-817

30. Kooistra T, Bosma PJ, Tons HAM, Van Den Berg AP, Meyer P, Princen HMG (1989) Plasminogen activator inhibitor 1: biosynthesis and mRNA level are increased by insulin in cultured human hepatocytes. Thromb Haemostas 62:723-728

31. Samad F, Yamamoto K, Loskutoff DJ (1996) Distribution and regulation of plasminogen activator inhibitor type- 1 in murine adipose tissue in vivo. J Clin Invest 97:37-46

32. Torr-Brown SR, Sobel, BE (1994) Plasminogen activator inhibitor is elevated in plasma and diminished in platelets in patients with diabetes mellitus. Thromb Res 75:473-477

33. Nordt TK, Sawa H, Fujii S, Sobel BE (1995) Augmentation of plasminogen ativator inhibitor type 1 (PAI-1) by proinsulin and insulin in vivo. Circulation 91:764-770

34. Schneider DJ, Absher PM, Ricci MA (1997) The dependence of augmentation of arterial endothelial cell expression of plasminogen activator inhibitor type 1 by insulin on soluble factors released from vascular smooth muscle cells. Circulation (in press)

35. Gebara OC, Mittleman MA, Sutherland P, et al. (1995) Association between increased estrogen status and increased fibrinolytic potential in the Framingham Offspring Study. Circulation 91:1952-1958 